\title{
DSP-based Synchronization Algorithm Implementation for OFDM
}

\author{
Mahmood A.K. Abdulsattar \\ Department of Electrical Engineering \\ University of Baghdad, Baghdad, Iraq
}

\author{
Raed Sattar Jebur \\ Department of Electrical Engineering \\ University of Baghdad, Baghdad, Iraq
}

\begin{abstract}
Synchronization has been one of the most important research topics in Orthogonal Frequency Division Multiplexing (OFDM) system because of its great sensitivity towards timing and frequency offset errors. This paper describes a real-time implementation of a timing and frequency synchronization for OFDM system using MATLAB software Simulink, DSP processor TMS320C6713 and Code Composer Studio (CCS). The hardware interface converts the baseband signal from the PC to the DSP Processor. Basic transmission and reception performances are evaluated in real time using Real Time data Transfer (RTDX). The practical results and performance evaluation of the synchronization algorithms in OFDM system is presented and discussed. The results are plotted for different offsets of CFO and STO with different values of signal to noise ratio. Also the bit error rate (BER) and the mean square error (MSE) of the system is analyzed.
\end{abstract}

\section{Keywords}

OFDM, Synchronization, MATLAB, Simulink, DSP.

\section{INTRODUCTION}

In [1], the rapidly growing of telecommunication market in the last decades create a need for new techniques that can provide high data rates. Many digital communications services have been deployed with constantly growing data rates like digital radio, digital television, and mobile Internet. The Orthogonal Frequency Division Multiplexing (OFDM) technology is able to provide a high transmission data rate with enhanced communication performance at a relatively small bandwidth cost, together with proper estimation and compensation of channel effects. Therefore, it has been widely applied in many wireless and mobile networks. Although the principles of OFDM modulation have been in existence since 1960 , in the last years OFDM modulation is appeared as a key modulation technique of commercial high speed communication systems. The principal reason of this increasing interest is due to its capability to provide highspeed data rate transmissions with low complexity and to counteract the Inter Symbol Interference (ISI) introduced by dispersive channels.

On the other hand, the use of OFDM systems with a high number of subcarriers has some drawbacks. One of the major drawbacks of OFDM is that it is more sensitive to synchronization errors. The performance of OFDM systems is particularly dependent on the synchronization error which occurs due to the Carrier Frequency Offset (CFO) and the Symbol Timing Offset (STO). The frequency mismatch between transmitter and receiver causes loss of orthogonally among the subcarriers giving rise to Inter Carrier Interference (ICI) which results in Signal to Noise Ratio (SNR) loss of the useful signal. Incorrect symbol timing brings signals from adjacent frames into the target frame resulting in severe ISI. Therefore, synchronization is very important to OFDM systems [2], [3].
In this paper, we study the DSP implementation of synchronization algorithm for OFDM system by using MATLAB/SIMULINK to design the model and then use the Embedded Target for TI C6000 DSP and Real-Time Workshop, to generate ANSI C code targeted to the Texas Instruments DSP development boards.

\section{OFDM SYNCHRONIZATION}

Synchronization has been one of the fundamental research topics in OFDM system because of its sensitivity to the timing and frequency errors [4]. In fact, STO and CFO can significantly weaken the performance of OFDM systems. Specifically, incorrect timing synchronization can cause interference between successive symbols and, if not perfectly compensated before the equalization process, can lead to severe performance degradation. In addition, a CFO induces an amplitude reduction of the useful signal and provokes interference between adjacent subcarriers [5].

\subsection{Timing Offset Error}

In an OFDM system, the IFFT and FFT are the fundamental functions used for the modulation and demodulation of OFDM symbol at transmitter and receiver respectively. In order to demodulate an OFDM symbol correctly at the receiver using N-point FFT, it is very much required to take exact samples of transmitted OFDM symbol. The correct starting point of FFT window is required to preserve the orthogonality among the sub-carriers. There is lot of advantages of OFDM system over single carrier system however all these advantages can be useful only when the orthogonality among sub-carriers is maintained. If one FFT window takes sample of two different OFDM symbol then it will generate ICI and ISI as shown in next section. Hence it can be stated that the FFT window timing synchronization in OFDM system is corresponds to symbol timing synchronization in single carrier transmission system [6].

Depending on the location of the estimated starting point of OFDM symbol, the effect of STO might be different. Figure 1 shows four different cases of timing offset, in which the estimated starting point is exact, a little earlier, too early, or a little later than the exact timing instance. Here, we assume that the multi-path delay spread incurs the lagged channel response of $\tau_{\max }$. In the current analysis, the effects of the noise and channel are ignored. Referring to Figure 1, let us discuss the effects of STO for these four different cases below.

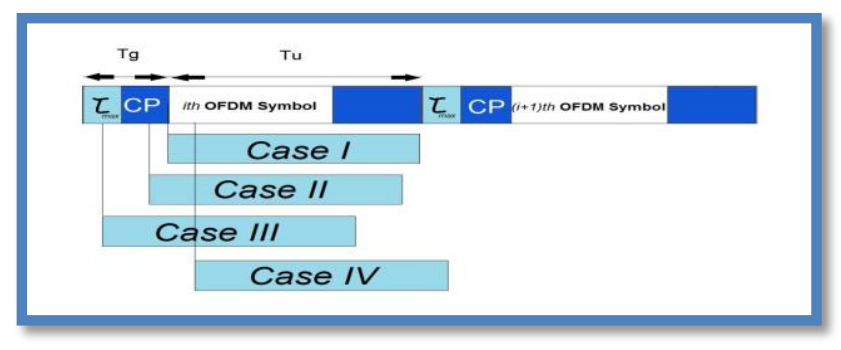

Figure 1: Four different cases of STO [6]. 
Case-I: This is the case when the estimated starting point of OFDM symbol coincides with the exact timing, preserving the orthogonality among sub-carriers, therefore the OFDM symbol can be perfectly recovered without any type of interference.

Case-II: This is the case when the estimated starting point of OFDM symbol is before the exact point, yet after the end of the (lagged) channel response to the previous OFDM symbol. In this case, the $i^{\text {th }}$ symbol is not overlapped with the previous $(i-1)^{t h}$ OFDM symbol, that is, without incurring any ISI by the previous symbol in this case.

Case-III: This is the case when the starting point of the OFDM symbol is estimated to exist prior to the end of the (lagged) channel response to the previous OFDM symbol, and thus, the symbol timing is too early to avoid the ISI. In this case, the orthogonality among subcarrier components is destroyed by the ISI (from the previous symbol) and furthermore, ICI occurs.

Case-IV: This is the case when the starting point of the OFDM symbol is estimated just after the exact point. In this case, the samples for current FFT operation interval is consists of a part of the current OFDM symbol $x(i, n)$ and a part of next symbol $x(i+1, n)$.

\subsection{Carrier Frequency Offset}

Synchronization is one of the major concerns of OFDM system. Synchronization includes both timing and frequency offset estimation and correction. The timing synchronization analysis has been done in the previous. Now we will analyze the effect of CFO and different methods of CFO estimation.

The baseband transmit signal is converted up to the passband by a carrier modulation and then, converted down to the baseband by using a local carrier signal of the same carrier frequency at the receiver. In general, there are two types of distortion associated with the carrier signal [6]. One is the phase noise due to the instability of carrier signal generators used at the transmitter and receiver, which can be modeled as a zero-mean Wiener random process. The other is the CFO caused by Doppler frequency shift $f_{d}$. Furthermore, even if we intend to generate exactly the same carrier frequencies in the transmitter and receiver, there may be an unavoidable difference between them due to the physically inherent nature of the oscillators. Let $f_{c}$ and $f_{c}^{\prime}$ denote the carrier frequencies in the transmitter and receiver, respectively. Let $f_{\text {offset }}$ denote their difference (i.e., $f_{\text {of } f s e t}=f_{c}-f_{c}^{\prime}$ ). Meanwhile, Doppler frequency $f_{d}$ is determined by the carrier frequency $f_{c}$ and the velocity $v$ of the receiver as

$f_{d}=\frac{v \cdot f_{c}}{c}$

where $\mathrm{c}$ is the speed of light. Let us define the normalized $\mathrm{CFO}, \epsilon$, as a ratio of the $\mathrm{CFO}$ to subcarrier spacing $\Delta \mathrm{f}$, shown as

$\epsilon=\frac{f_{\text {of } f s e t}}{\Delta f}$

In OFDM system, there is a stringent requirement of carrier frequency synchronization. The whole concept of OFDM and all other advantages are depending on the orthogonality of sub-carriers. If the orthogonality between sub-carriers lost due to CFO, then the performance of OFDM system degrades. CFO causes a number of impairments including attenuation and rotation of each of the subcarriers and ICI between subcarriers.

\section{SYNCHRONIZATION ALGORITHMS}

Synchronization has been one of the important research topics in OFDM system because of its sensitivity to the timing and frequency offsets. So it became a topic of interest for many researchers, and they were published proposing different synchronization techniques as solutions for the synchronization problem in OFDM systems. The OFDM synchronization can be divided into data-aided and non-dataaided categories. The data-aided category uses a training sequence or pilot symbols for estimation. It has high accuracy and low calculation, but loses the bandwidth and reduces the data transmission speed. The non-data aided category often uses the cyclic prefix correlation. It doesn't waste bandwidth and reduce the transmission speed, but its estimation range is too small, not suitable for acquisition. Some of these papers proposed for both categories are listed below:

Paul H. Moose (1994) [7], he discusses the effects of frequency offset on the performance OFDM system. A Maximum Likelihood Estimation (MLE) algorithm is derived and its performance computed and compared with simulation report. A strategy is described for initial acquisition in the event of uncertainty in the initial offset that exceeds $1 / 2$ the carrier spacing, the limit of the MLE algorithm.

Jan-Jaap van de Beek, Magnus Sandell, and Per Ola Borjesson (1997) [8], present the joint Maximum Likelihood (ML) symbol-time and carrier-frequency offset estimator in OFDM systems. Redundant information contained within the cyclic prefix enables this estimation without additional pilots. Simulations show that the frequency estimator may be used in a tracking mode and the time estimator in an acquisition mode.

Timothy M. Schmidl and Donald C. Cox (1997) [9], a rapid synchronization method is presented for an orthogonal frequency-division multiplexing (OFDM) system using either a continuous transmission or a burst operation over a frequency-selective channel. By averaging over all the subchannels, it works well in frequency selective channels. This method also gives very accurate estimates of symbol timing and carrier frequency offset and provides a very wide acquisition range for the carrier frequency offset.

In March 1999, Michele Morelli and Umberto Mengali (1999) [10], An efficient algorithm has been proposed by Schmidl and Cox for carrier frequency estimation in orthogonal frequency-division multiplexing systems. The scheme is based on the transmission of a training symbol composed of two identical halves in the time domain. In this paper we extend this algorithm by considering a training symbol composed of $L>2$ identical parts. This makes it possible to achieve a better accuracy at the cost of some increase in computational load.

They have presented and analyzed technique for OFDM synchronization based on pseudo noise sequence preamble makes it possible to sum received sample coherently before multiplying them. In this way it is possible to decrease the influence from noise or interference when the timing is wrong. A lower detection threshold can therefore be used, which results in better detection performance, i.e. a lower false detection probability and a lower probability of missing the sync signal.

Fredrik Tufvesson, Ove Edfors and Mike Faulkner (1999) [11], They have presented and analyzed technique for OFDM synchronization based on pseudo noise sequence preamble makes it possible to sum received sample coherently before 
multiplying them. In this way it is possible to decrease the influence from noise or interference when the timing is wrong. A lower detection threshold can therefore be used, which results in better detection performance, i.e. a lower false detection probability and a lower probability of missing the sync signal. Synchronization based on PN-sequence preambles offered greater power reductions in stand by model.

H. Minn, M. Zeng, and V. K. Bhargava (2000) [12], two timing offset estimation methods for OFDM systems are presented as modifications to Schmidl and Cox's method where a training symbol containing two identical halves is used. The first method uses two modifications: 1) all samples over one symbol period (excluding guard interval), instead of over half symbol period, are used in calculation of half symbol energy required in timing metric and 2) averaging of timing metrics over a window of guard interval length is used instead of $90 \%$ maximum points averaging. The second method uses a training symbol containing four equal length parts: the first two are identical and the last two are the negative of the first two.

Various techniques have been proposed in the literature for combined timing and frequency synchronization in OFDM. In this paper, we focus on non data-aided methods which can be applied to OFDM system. Time domain synchronization is achieved through a joint ML symbol time and CFO estimator through the redundant information contained in the $\mathrm{CP}$.

The Synchronization algorithm that is implemented is presented in [8]. The time and frequency offsets are estimated simultaneously using the correlation introduced by the cyclic prefix.

\subsection{Van de Beek Algorithm [8]}

Exploiting the redundancy created by the $\mathrm{CP}$, we can estimate time and frequency parameters. This is most commonly done by averaging the correlation of the $\mathrm{CP}$ and the end of the OFDM symbol.

The subsystem presented here is based on the algorithms developed by Van de Beek and et al [8]. They exploit the CP by correlating it with a delayed version of itself. When the repeated pattern is located, a peak is generated in order to detect the frame arrival and the phase between patterns gives the CFO.

In this algorithm $2 \mathrm{~N}+\mathrm{L}$ samples are observed as shown in Figure 2. In this interval, it is assumed that there is one complete OFDM symbol with its cyclic prefix. If we define $\mathrm{r}(\mathrm{k})$ as the set of samples contained in the observed interval, $\theta$ as the index of the start of the symbol and $\epsilon$ as the frequency offset, we can find the maximum likelihood estimate (MLE) of $\theta$ and $\epsilon$ by maximizing their log-likelihood function, which is defined as the logarithm of the probability density function $\mathrm{f}(\mathrm{r} \mid \theta, \epsilon)$ of the $2 \mathrm{~N}+\mathrm{L}$ samples in $\mathrm{r}(\mathrm{k})$ given $\theta$ and $\epsilon$.

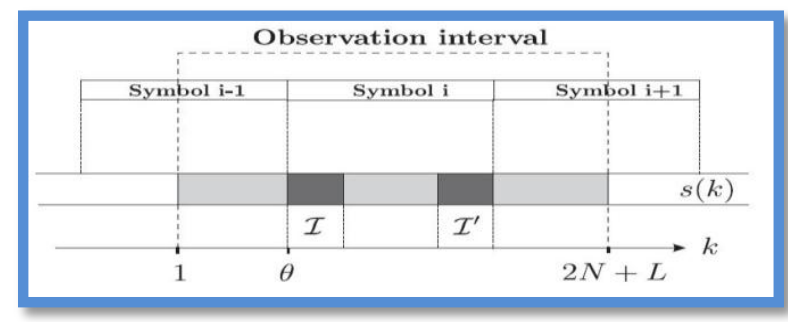

Figure 2: Structure of OFDM signal with cyclically extended symbols.
The joint ML estimation becomes

$\theta_{M L}=\arg \max \{|\gamma(\theta)|-\rho \emptyset(\theta)\}$

$\epsilon_{M L}=-\frac{1}{2 \pi} \operatorname{angle}\left(\gamma\left(\theta_{M L}\right)\right)$

Where

$\gamma(m)=\sum_{k=m}^{m+L-1} r_{k} r_{k+N}^{*}$

$\emptyset(m)=0.5 \sum_{k=m}^{m+L-1}\left|r_{k}\right|^{2}+\left|r_{k+N}\right|^{2}$

$\rho=\frac{S N R}{S N R+1}$

In Figure 3 we can see the scheme for this synchronization method.

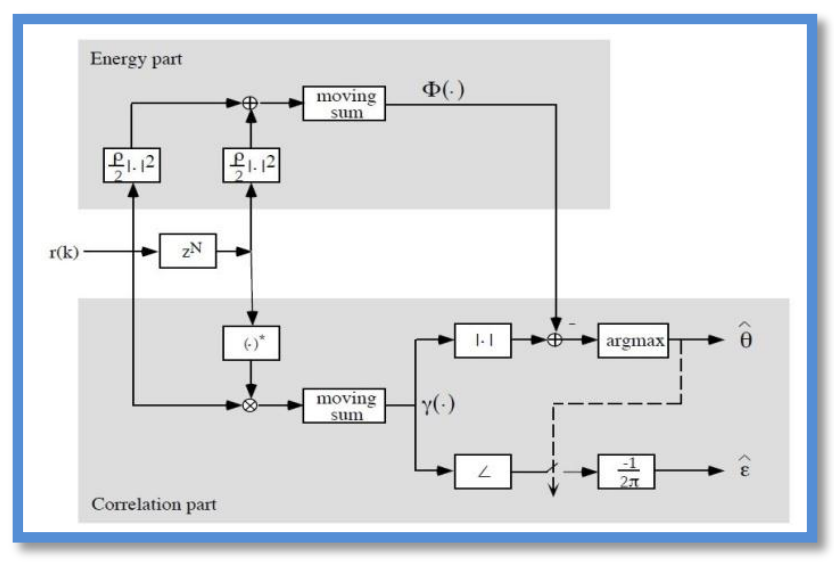

Figure 3: Structure of the estimator.

The algorithm consists of two main branches. The top one calculates an energy term. While the bottom one calculates the correlation term required for estimating both symbol arrival time and phase offset. Equation (6) shows the calculation of the energy term and Equation (5) shows the calculation of the correlation term. The factor $\rho$ is the magnitude of the correlation coefficient between $r_{k}$ and $r_{k+N}$; it depends on the signal-to-noise ratio but can be set to 1 . Figure 4 shows the ML estimation results for both time and frequency estimation.

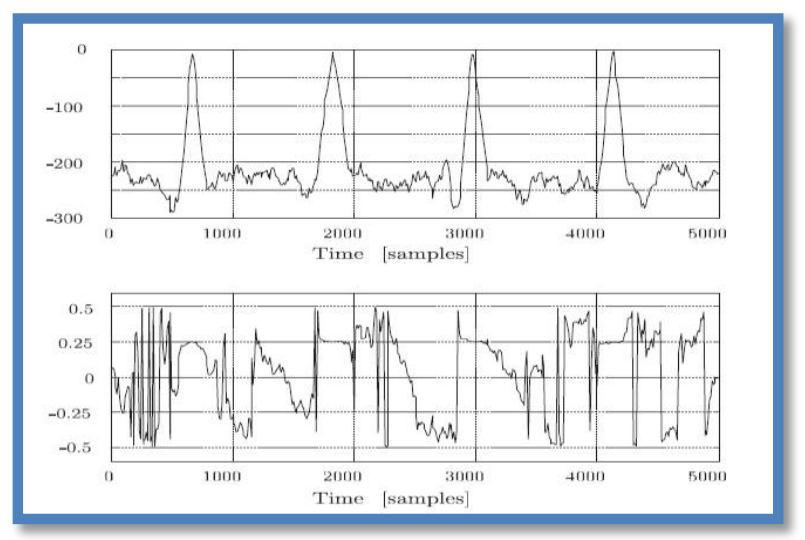

Figure 4: The signals that generate the ML-estimates $(\mathrm{N}=$ $1024, L=128, \epsilon=0.25$ and $S N R=15 \mathrm{~dB}$ ).

The maximizing indices (top) yield the time estimates $\theta_{M L}$. At these time instants the arguments of $\gamma(\theta)$ (bottom) yield $\epsilon_{M L}$. 


\section{DIGITAL SIGNAL PROCESSING}

DSP is one of the fastest growing fields of technology and computer science in the world. In today's world almost everyone uses DSPs in their everyday life but, unlike PC users, almost no one knows that he/she is using DSPs. DSPs are special purpose microprocessors used in all kind of electronic products, from mobile phones, modems and CD players to the automotive industry; medical imaging systems to the electronic battlefield and from dish washers to satellites [13].

There are many reasons why we process these analog signals in the digital world. Traditional signal processing was achieved by using analogue components such as resistors, capacitors and inductors. However, the inherent tolerance associated with these components, temperature and voltage changes and mechanical vibrations can dramatically affect the effectiveness of analogue circuitry. On the other hand, DSP is inherently stable, reliable and repeatable. With DSP it is easy to chance, correct or updates applications. Additionally, DSP reduces noise susceptibility, chip count, development time, cost and power consumption [14].

\subsection{TMS320C6713 DSP Description [15], [16]}

The TMS320C67 DSPs (including the TMS320C6713 device) compose the floating point DSP generation in the TMS320C6000 DSP platform. The TMS320C6713 (C6713) device is based on the high-performance, Very Long Instruction Word (VLIW) architecture developed by Texas Instruments, making this DSP an excellent choice for multichannel and multifunction applications.

The features of the C6713, $225 \mathrm{MHz}$ device delivering up to1800 MIPs. This DSP generation is designed for applications that require high precision accuracy. The C6713 is based on the TMS320C6000 DSP platform designed to fit the needs of high-performing high-precision applications such as pro-audio, medical and diagnostic. Other hardware features of the C6713 board include:

$\begin{array}{ll}\text { - } & \text { Embedded JTAG support via USB } \\ \text { - } & \text { High-quality 24-bit stereo codec } \\ & \text { Four 3.5mm audio jacks for microphone, line in, } \\ \text { - } & \text { speaker and line out } \\ \text { - } & \text { Expansion port connector for plug-in modules } \\ \text { - } & \text { On-board standard IEEE JTAG interface } \\ \text { - } & +5 \mathrm{~V} \text { universal power supply }\end{array}$

The DSP environment used in this paper is the C6713. Figure 5 shows the architecture of the C6713.

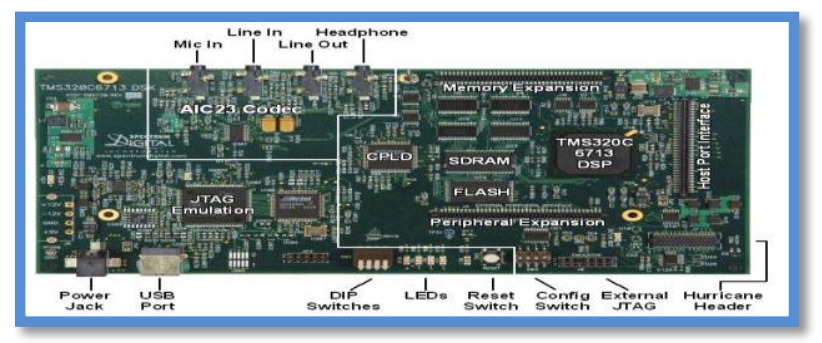

Figure 5: TMS320C6713 board architecture [17], [18].

\section{DSP IMPLEMENTATION}

In the past, the small systems implemented on DSP by writing its algorithm by $\mathrm{C} / \mathrm{C}++$ or assembly, using a text programming languages in writing, but long and complicated algorithms makes the process exhaustive and difficult to debug, modify, and learn [40].

The following sections describe the simulation and implementation of OFDM transceiver with synchronization algorithm on a DSP platform through a graphical programming language, which is SIMULINK. This makes every part in the system architecture very clear and easier to understand, follow, modify and debug.

\subsection{DSP Software Implementation}

In [19], the C6713 board, like a personal computer, needs to load software to establish its behavior and function. This software's can be designed in a variety of approaches. The design approach that the real time implementation will follow is illustrated in Figure 6 . The designer will begin with a concept of what they want to program. The next step is to model the concept with blocks from Simulink's large collection of pre written block sets. Basically, a block diagram that models the concept is built using Simulink. If a specific block required is not included in Simulink's block sets, the designer may choose to write their own blocks from scratch using MATLAB. At this point the design is still not designed to operate on any specified hardware. To do that, the designer uses the C6x Target and RTW to generate (or build) ANSI C code intended for the C6713. The C6x Target will then automatically take the generated ANSI $\mathrm{C}$ code and uses the Code Composer Studio (CCS) tools to compile specific machine code and finally loads the targeted machine code to the C6713 hardware. For the experienced designer, they may choose to directly write the ANSI C code.

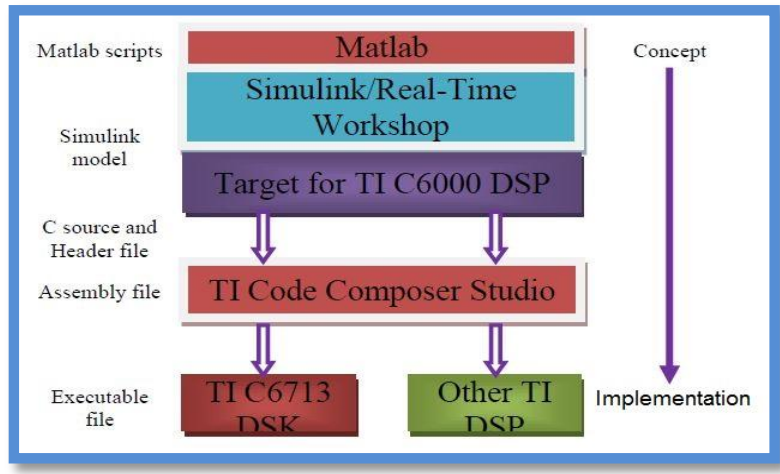

Figure 6: Software design flow.

\subsubsection{Simulation Model}

OFDM transmitter with variable symbol timing and carrier frequency error is designed. The model implements a 64 carrier OFDM waveform with a 16 point cyclic prefix. The OFDM symbol has 16 pilots, and uses 4 Quadrature Amplitude (QAM) for the 48 remaining data streams.

Once the implementation of the model in SIMULINK is achieved , and already tested that it works properly, the next step is targeting the model on the DSP board. In this paper, the implementation of the model on a TMS320C6713 DSP board from Texas Instrument is carried out. Having the implementation of the model in Simulink and after testing that this is working properly, the next step is targeting the model on the DSP board. For accomplishing this assignment, the following tools have been used: 
- Code Composer Studio v 3.1

- Embedded Target for TI c6000 DSP

\subsection{The Code Composer Studio [20], [21]}

CCS is the necessary software support tools, which provides an integrated development environment (IDE), bringing together the $\mathrm{C}$ compiler, assembler, linker, debugger, and so on. It has graphical capabilities and supports real-time debugging. It provides an easy-to-use software tool to build and debug programs.

The $\mathrm{C}$ compiler compiles a $\mathrm{C}$ source program to produce an assembly source file. The assembler assembles the source file to produce a machine language object file, combines object files and object libraries as input to produce an executable file by the linker with extension ".out". This executable file can be loaded and run directly on the C6713 processor.

\subsection{Real-Time Data Exchange}

DSP programs can be transfer data between the DSP device and a host computer without affecting the DSP application using RTDX. There are components on the DSP device (target) and the host computer that allow this communication to happen [22].

Real-Time Data Exchange (RTDX) provides real-time, continuous visibility into the way target applications operate in the real world. RTDX allows system developers to transfer data between target devices and a host without interfering with the target application. Key statistics and performance can be monitored in real time. Through the Joint Team Action Group (JTAG), communication with on-chip emulation support occurs to control and monitor program execution. The C6713 DSK board includes a JTAG interface through the USB port.

\subsection{Target for c6000 DSP [19], [23]}

Embedded Target for the TMS320C6000 DSP Platform integrates Simulink ${ }^{\circledR}$ and MATLAB $®$ with Texas Instruments eXpressDSPTM tools. The software collection lets you develop and validate digital signal processing designs from concept through code. The Embedded Target for C6000 DSP consists of the C6000 target that automates rapid prototyping on your C6000 hardware targets. The target uses C code generated by Real-Time Workshop ${ }^{\circledR}$ and your development tools to build an executable file for your targeted processor. The Real-Time Workshop build process loads the targeted machine code to your board and runs the executable file on the digital signal processor. Using the Embedded Target for C6000 DSP and Real-Time Workshop, you can create executable code for the following boards:

-
-
-
C67676 DSP from Texas Instruments
C6713 DSP from Texas Instruments (board used in
this paper work)
TMDX326040. A daughter card for the C6711 DSK

\section{SIMULATION AND DSP BOARD RESULTS}

The simulation and DSP results are taken for $\mathrm{CFO}=0 \mathrm{~Hz}, 20$ $\mathrm{Hz}, 100 \mathrm{~Hz}, 300 \mathrm{~Hz}, 500 \mathrm{~Hz}$, while STO = 0 sample, 1 sample, 4 samples and $\mathrm{SNR}=10 \mathrm{~dB}$. Then the Bit Error Rate (BER) and Mean Square Error (MSE) are plotted against SNR.

\subsection{Symbol Timing Offset and Carrier Frequency Offset effect}

These are some of simulation and DSP cases results.

Case 1: There is no timing and frequency error $\mathrm{CFO}=0, \mathrm{STO}$ $=0$, Channel $=$ No Noise

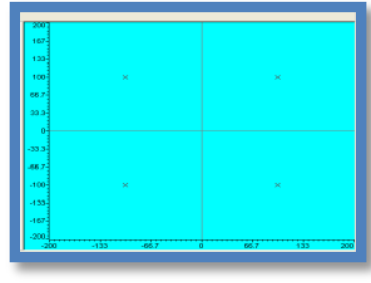

(a)Data(MATLAB)

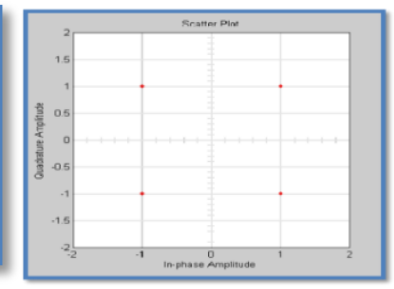

(b)Data(C6713)
Figure 7: MATLAB and DSP data Constellation provided $\mathrm{CFO}=\mathbf{0}, \mathrm{STO}=\mathrm{O}$ and No Noise.

Case 2: In this case $\mathrm{STO}=1$ sample, $\mathrm{CFO}=0$ and Channel $=$ No Noise.

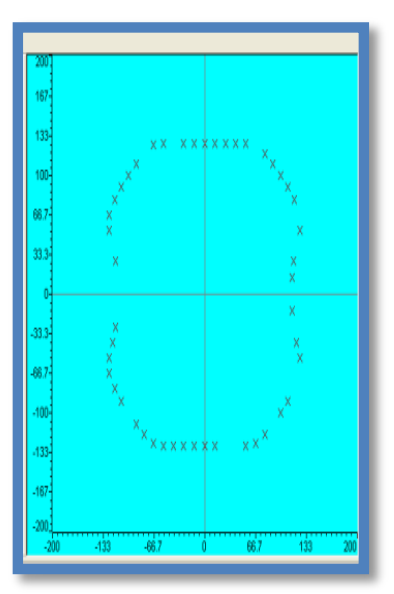

(a) Data (MATLAB)

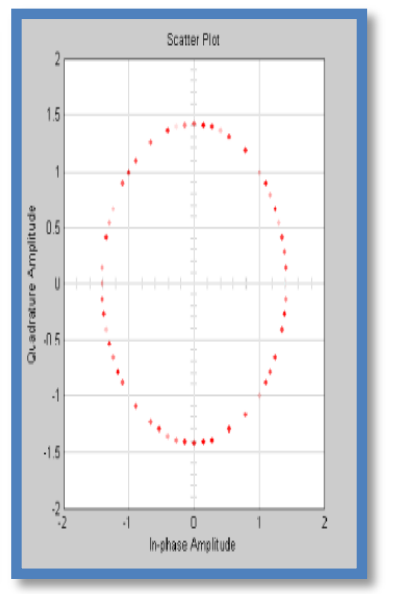

(b) Data (C6713)
Figure 8: MATLAB and DSP data Constellation provided $\mathrm{CFO}=0, \mathrm{STO}=1$ sample and No Noise

Case 3: In this case $\mathrm{STO}=0, \mathrm{CFO}=100 \mathrm{~Hz}$, and Channel $=$ No Noise.

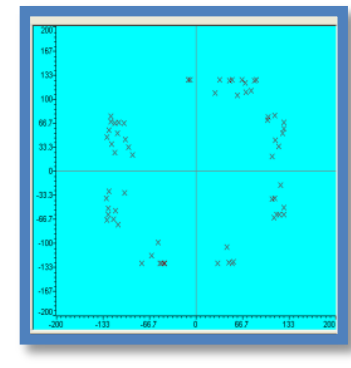

(a) Data (MATLAB)

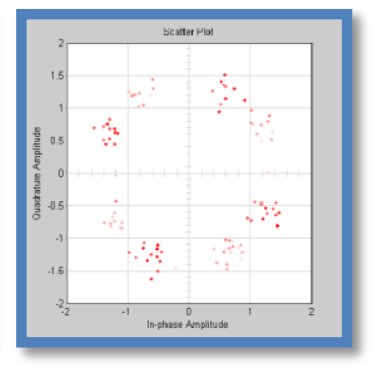

(b) Data (C6713)
Figure 9: MATLAB and DSP data Constellation provided $\mathrm{CFO}=20 \mathrm{~Hz}, \mathrm{STO}=0$ and Channel $=$ No Noise.

Case 17: In this case $\mathrm{STO}=0, \mathrm{CFO}=100 \mathrm{~Hz}$ and Channel $=$ AWGN SNR $=10$. 


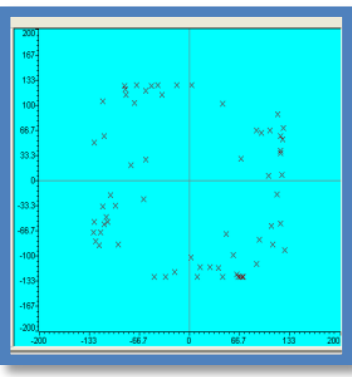

(a) Data (MATLAB)

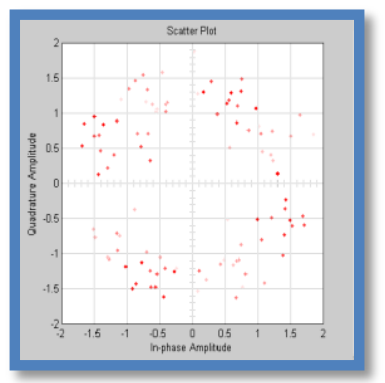

(b) Data (C6713)
Figure 10: MATLAB and DSP data Constellation provided $\mathrm{CFO}=100 \mathrm{~Hz}, \quad$ STO $=0$ and Channel $=\mathrm{AWGN}$ $\mathrm{SNR}=10$

After simulate the model on different values of STO and CFO and use Monte Carlo Simulink, plot the Bit Error Rate and SNR relation for these different values.

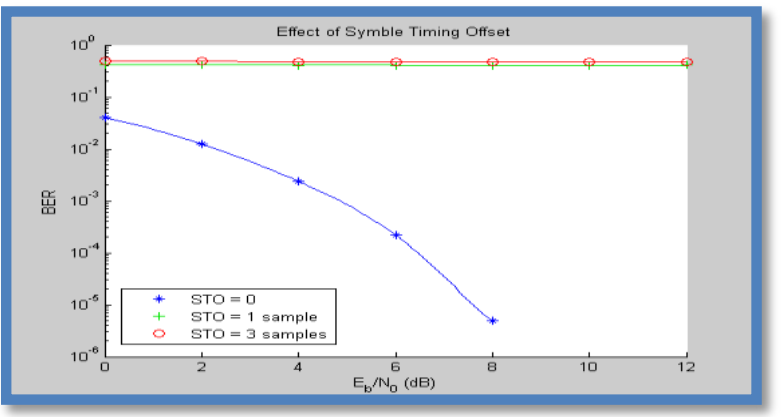

Figure 11: Illustrates the Effect of Symbol Timing Offset.

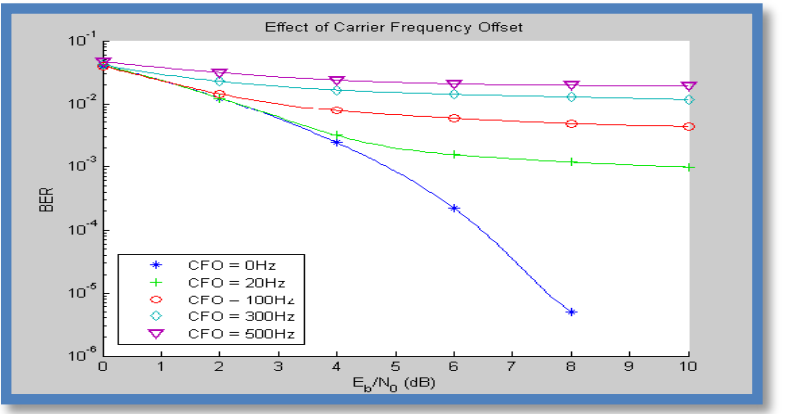

Figure 12: Illustrates the Effect of Carrier Frequency Offset.

From MATLAB results, the Mean Square Error with respect to SNR for different values of STO and CFO had been plotted after record the input data and the output data, calculate the mean square error for these results. Figure 13 and Figure 14 illustrate the effect of timing and frequency offsets.

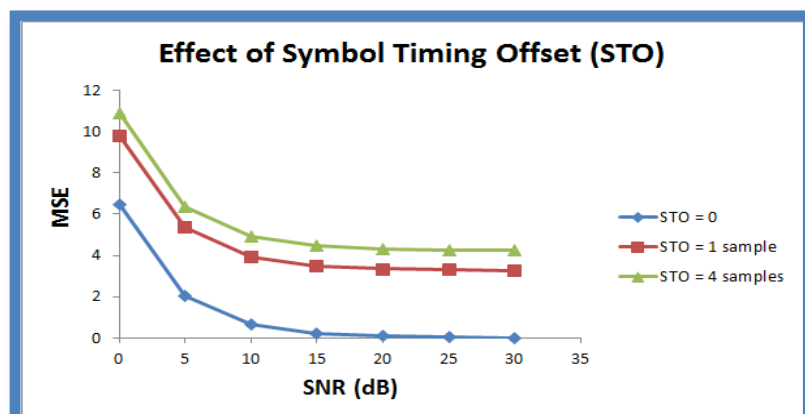

Figure 13: Illustrates the Effect of Symbol Timing Offset.

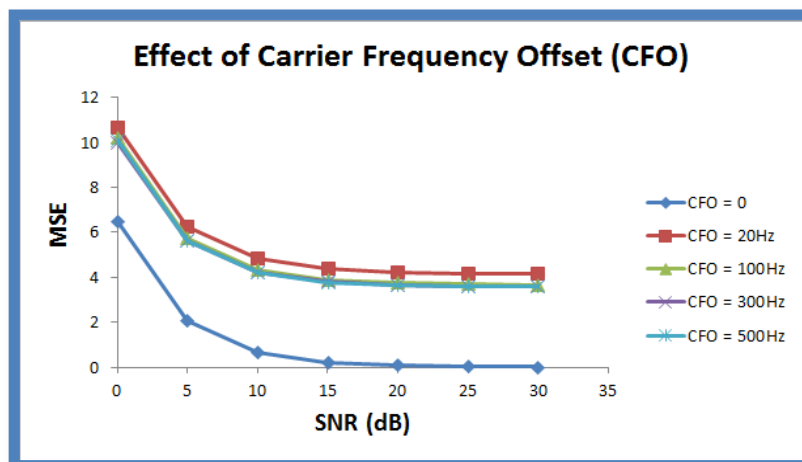

Figure 14: Illustrates the Effect of Carrier Frequency Offset.

\subsubsection{Synchronization Performance Analysis}

When perform the synchronization algorithm, the ML estimation result have voltage peaks in the time shown in the top part of Figures 15, 16 that correspond to best synchronized frame. This is because the result of correlation of the cyclic prefix with its delayed version is maximized when they are coinciding together. These peaks in the time (best synchronization) means the frequency offset is corrected to the minimum possible value as shown in the down part of Figures 15,16 .

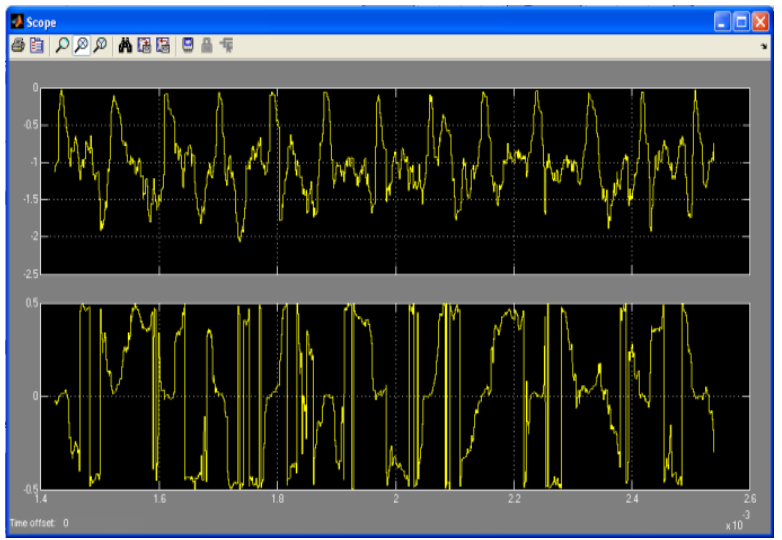

Figure 15: ML estimation Simulink result (MATLAB). 


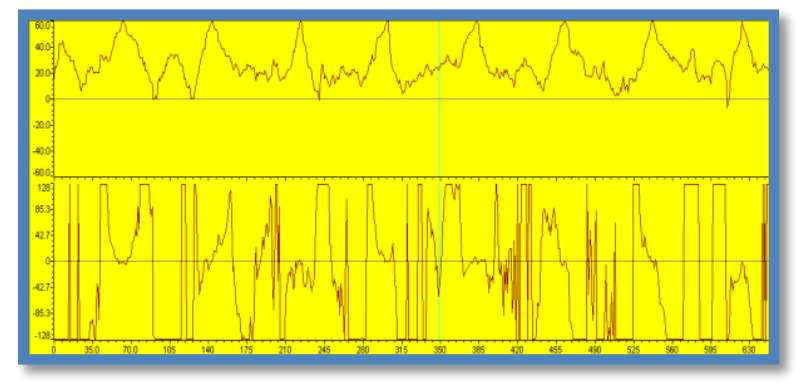

Figure 16: ML estimation DSP result (C6713).

The RTDX was used to transmit digital data to and from the Texas Instruments C6713 DSK board. To utilize this tool, an interface was needed to specify what data would be transmitted. Graphical User Interface from MATLAB software use to design GUI form to send and receive data from host to target and from target to host. The results are shown in Figure 17.

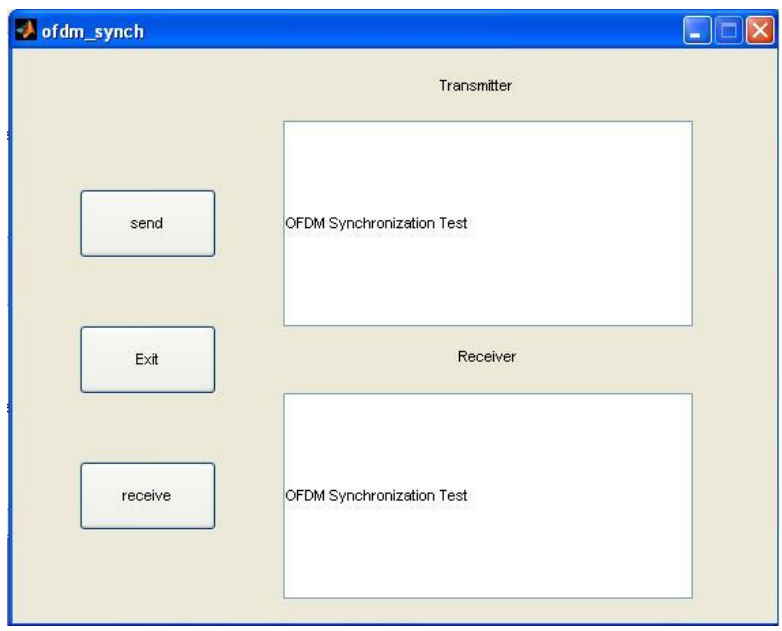

Figure 17: GUI Form for the RTDX interface illustrates the read and writes from and to DSP board.

The code from the embedded target for C6000 DSP and RealTime Workshop is generated. This can create executable code for this board. We opened RTDX program that we designed before and write a message in the transmitter text box and then press "send" bottom. The software send data from host (PC) to target (board) then wait few second to process the data. Then we can press "receive" bottom to receive data from target to host.

\section{CONCLUSIONS}

This paper has two primary purposes. First, it studied and investigated the need for timing and frequency synchronization errors in OFDM system, analyzed how big an impact of time and frequency offsets could be to an OFDM system, and discussed techniques to combat those offsets. The paper focuses primarily on non data-aided technique. It doesn't waste bandwidth and reduce the transmission speed, but its estimation range is too small, not suitable for acquisition. The performance of the synchronization technique is analyzed mainly through software simulation.

Second, it implemented this OFDM system with synchronization algorithm on a TMS320C6713 DSP board. The real-time code for the complete system is automatically generated using Embedded Target and real-time Work Shop. No hand-coding is required. Time for implementation and testing is minimized.
Real-time Workshop is powerful and applicable tool that enables automatic $\mathrm{C}$ code generation from the Simulink model. Generated code is well optimized and it's comparable with the hand written code. Superior property of the Simulink and the RTW is its open architecture that enables adding custom blocks to the Simulink and custom target development.

The Real Time Data Exchange function creates a link between the DSP and the computer. Through this link data can be exchanged while the program is running by using GUI software to read and write the data to show the results.

The Van de Beek algorithm is found to be robust for both CFO and STO simultaneously. It can handle offset of about $\mathrm{CFO}=500 \mathrm{~Hz}$ and $\quad$ STO $=4$ samples simultaneously without wasting extra. This is advantageous over the standard 802.11a OFDM where the overall data rate is lowered due to using the training sequence.

\section{REFERENCES}

[1] Manushree Bhardwaj, Arun Gangwar, Devendra Soni, "A Review OFDM: Concept, Scope \& its Applications," IOSR Journal of Mechanical and Civil Engineering (IOSRJMCE), ISSN : 2278-1684 Volume 1, Issue 1 (MayJune 2012), PP 07-11.

[2] Anandh Leno.D, Arul Rex.A, "Design of Resource Efficient Low Power Correlator for Communication," International Journal of Research in Advent Technology, Vol.2, No.2, February 2014

[3] Guest Editors:Heidi Steendam,Mounir Ghogho,Marco Luise, Erdal Panayirci, and Erchin Serpedin, "Synchronization inWireless Communications," EURASIP Journal on Wireless Communications and Networking, Hindawi Publishing Corporation 2009.

[4] T. Pollet, M. Van Bladel, and M. Moeneclaey, "Ber sensitivity of OFDM systems to carrier frequency offsetand wiener phase noise," IEEE Trans. Commun., vol. 43, pp. 191-193, Feb./Mar/Apr1995.

[5] Bo Ai, Zhi-xing Yang, Chang-yong Pan, Jian-hua Ge, Yong Wang, and Zhen Lu, "On the Synchronization Techniques for Wireless OFDM Systems," IEEE Transactions On Broadcasting , Vol. 52, No. 2, June 2006.

[6] Yong Soo Cho, Jaekwon Kim, Won Young Yang, Chung G. Kang October 2010 MIMO-OFDM Wireless Communications with MATLAB".

[7] P. H. Moose, "A Technique for Orthogonal Frequency Division Multiplexing Frequency Offset Correction," IEEE Transactions on Communications, Vol. 42, 1994, pp. 2908-2914.

[8] J.-J. Van de Beek, M. Sandell and P. O. Börjesson, "ML Estimation of Time and Frequency Offset in OFDM Systems," IEEE Trans. Signal Processing, Vol. 45, 1997, pp. 1800-1805.

[9] T. M. Schmidl and D. C. Cox, "Robust Frequency and Timing Synchronization for OFDM," IEEE Trans. Commun., Vol. 45, 1997, pp. 1613-1621.

[10] M. Morelli and U. Mengali, "An Improved Frequency Offset Estimator for OFDM Applications," IEEE Communications Letters, Vol. 3,1999, pp. 75-77. 
[11] F. Tufvesson, O. Edfors and M. Faulkner, "Time and Frequency Synchronization for OFDM using PNsequence Preamble," in Proc. Veh. Technol. Conf., Vol. 4, 1999, pp. 2203-2207.

[12] H. Minn, M. Zeng and V. K. Bhargava, "On Timing Offset Estimation for OFDM Systems," IEEE Commun. Letters, Vol. 4, 2000, pp. 242-244.

[13] Özlem Kalinli. "Signal Processing with DSPs". Undergraduation Thesis. June-2001.

[14] B. Preetham Kumar, "DIGITAL SIGNAL PROCESSING LABORATORY," A CRC title, part of the Taylor \& Francis imprint, 2005 by CRC Press.

[15] Milind U. Nemade, Satish K.Shah, " Real Time Speech Recognition Using DSK TMS320C6713,” International Journal of Advanced Research in Computer Science and Software Engineering, Volume 4, Issue 1, January 2014.

[16] Texas Instrument Inc., "TMS320C6713 Floting-Point Digital Signal Processor" SPRS186L ,November 2005.

[17] Azeddine Wahbi, Ahmed Roukhe, Laamari Hlou," Conception and Real Time DSK C6713 of a Low Cost Adaptive Acoustic Noise Cancellation (ANC) Based Fast Fourier Transform (FFT) and Circular Convolution for Improving the Quality of Voice Communications,"
Int.J.Computer Technology \& Applications, Vol 5 (2),630-639, March-April 2014

[18] Azeddine Wahbi, Ahmed Roukhe, Laamari Hlou, "Modeling and real-time DSK C6713 implementation of normalized least mean square (NLMS) adaptive algorithm for acoustic noise cancellation (ANC) in voice communications," Journal of Theoretical and Applied Information Technology, 20 Th July 2014. Vol. 65 No.2.

[19] R. Chassaing and D. Reay," Digital Signal Processing and Application with the TMS320C6713 and TMS320C6416 DSK" Second Edition, John Wily \& Sons, Inc., 2005.

[20] Nasser Kehtarnavaz and Namjin Kim, "Real-Time Digital Signal Processing Based on the TMS320C6000," Linacre House, Jordan Hill, Oxford OX2 8DP, UK 2005.

[21] Dr. Seema Verma, Pawan Sharma, "Hardware Implementation of OFDM system on TMS320C6713 and verifying the results by using DIP Switches and LEDs," IPASJ International Journal of Electronics \& Communication (IIJEC), Volume 1, Issue 6, December 2013.

[22] Embedded Target for the TI TMS320C6000 DSP Platform (For use with Real-Time Workshop (Version 1)). July 2002.

[23] 